\title{
ASYMPTOTICALLY LINEAR ELLIPTIC SYSTEMS WITH PARAMETERS
}

\author{
CHAOQUAN PENG \\ Department of Mathematics, South-Central University for Nationalities, Wuhan 430074, \\ The People's Republic of China \\ e-mail:pcq1979@163.com
}

(Received 27 May 2009; accepted 28 October 2009)

Abstract. In this paper, we show that the semi-linear elliptic systems of the form

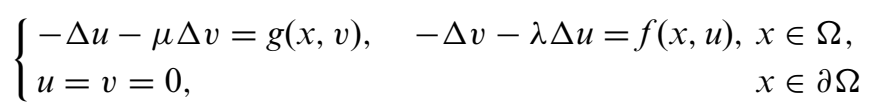

possess at least one non-trivial solution pair $(u, v) \in H_{0}^{1}(\Omega) \times H_{0}^{1}(\Omega)$, where $\Omega$ is a smooth bounded domain in $\mathbb{R}^{N}, \lambda$ and $\mu$ are non-negative numbers, $f(x, t)$ and $g(x, t)$ are continuous functions on $\Omega \times \mathbb{R}$ and asymptotically linear at infinity.

2000 Mathematics Subject Classification. AMS classification: 35J60, 35 J65.

1. Introduction. In this paper, we consider the existence of non-trivial solutions of non-linear elliptic systems

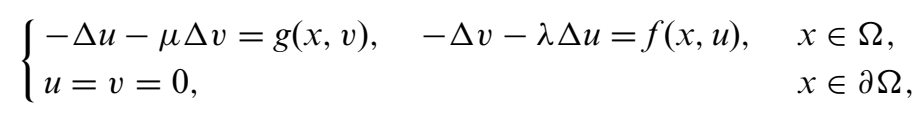

where $\Omega \subset \mathbb{R}^{N}$ is a smooth bounded domain, $\lambda$ and $\mu$ are non-negative numbers, $f(x, t)$ and $g(x, t)$ are continuous functions on $\Omega \times \mathbb{R}$ and asymptotically linear at infinity for $t$.

In the case of $\lambda=\mu=0$, in recent years, much attention has been paid to the existence of non-trivial solutions of problem (1.1) for the case that $f$ and $g$ are superlinear, see [1], [2], [3], [7] and references therein. In [4], G. Li and J.Yang considered the asymptotically linear elliptic systems

$$
-\Delta u+u=g(x, v), \quad-\Delta v+v=f(x, u), x \in \mathbb{R}^{N}
$$

it obtained a positive solution by using linking theorem under the Cerami compactness condition.

If $\lambda, \mu \neq 0$, the problem has some new features. First, by the Pohozaev-type identity, the parameters $\lambda$ and $\mu$ affect the sub-critical range of the growth of non-linear terms at infinity. Second, if $\lambda \mu<1$, the decomposition of the space in the framework involves the parameters, see [5, 6]. Moreover, $f$ and $g$ are superlinear in [5] and are asymptotically linear in [6].

In this paper, we will consider asymptotically linear elliptic systems (1.1) in $E=$ $H_{0}^{1}(\Omega) \times H_{0}^{1}(\Omega)$ with parameters $\lambda, \mu$ satisfies $\lambda \mu>1$, which allow us to define an 
equivalent norm on $E$. In fact, let $E$ be equipped with the norm

$$
\|z\|_{E}=\left(\int_{\Omega}\left(|\nabla u|^{2}+|\nabla v|^{2}\right) d x\right)^{\frac{1}{2}},
$$

where $z=(u, v)$. Since $\lambda \mu>1$, then there exists a real number $l>0$ such that $\lambda>l>\frac{1}{\mu}$ and we have

$$
\begin{aligned}
\max \left\{\frac{1+\lambda}{2}, \frac{1+\mu}{2}\right\}\left(|\nabla u|^{2}+|\nabla v|^{2}\right) & \geq \nabla u \nabla v+\frac{\lambda}{2}|\nabla u|^{2}+\frac{\mu}{2}|\nabla v|^{2} \\
& \geq \min \left\{\frac{\lambda-l}{2}, \frac{\mu}{2}-\frac{1}{2 l}\right\}\left(|\nabla u|^{2}+|\nabla v|^{2}\right) .
\end{aligned}
$$

Then we may introduce a new inner product on $E$ by the formula

$$
\langle(u, v),(\varphi, \psi)\rangle=\int_{\Omega}(\lambda \nabla u \nabla \varphi+\nabla u \nabla \psi+\nabla v \nabla \varphi+\mu \nabla v \nabla \psi) d x,
$$

and the corresponding norm is

$$
\|z\|=(\langle z, z\rangle)^{\frac{1}{2}}=\left(\int_{\Omega}\left(\lambda|\nabla u|^{2}+2 \nabla u \nabla v+\mu|\nabla v|^{2}\right) d x\right)^{\frac{1}{2}}, \forall z=(u, v) \in E .
$$

The norms $\|\cdot\|$ and $\|\cdot\|_{E}$ are then equivalent if $\lambda \mu>1$ by (1.2).

We assume that $f$ and $g$ satisfy

(H1) $f, g \in C^{1}(\Omega \times \mathbb{R}, \mathbb{R}), f(x, t)=g(x, t)=0$ if $t \leq 0$.

(H2) $\lim _{t \rightarrow 0}(f(x, t) / t)=\lim _{t \rightarrow 0}(g(x, t) / t)=0$ uniformly with respect to $x \in \Omega$ and $f(x, t)>0, g(x, t)>0$ for $t>0, x \in \Omega$.

(H3) $\lim _{t \rightarrow \infty}(f(x, t) / t)=l>0, \lim _{t \rightarrow \infty}(g(x, t) / t)=m>0$ uniformly in $x \in \Omega$.

(H4) $f(x, t) / t$ and $g(x, t) / t$ are non-decreasing in $t \geq 0$ for $x \in \Omega$.

Let $\lambda_{1}$ be the first eigenvalue of $\left(-\Delta, H_{0}^{1}(\Omega)\right)$ and $\varphi_{1}>0$ be the corresponding eigenfunction. Define $A=\min \left\{\frac{l}{1+\lambda}, \frac{m}{1+\mu}, \frac{m \lambda+\mu l-\sqrt{(m \lambda-\mu l)^{2}+4 m l}}{2(\lambda \mu-1)}\right\}$.

The main result of this paper is as follows:

TheOREM 1.1. Suppose $(H 1)-(H 4)$ hold. If $\lambda \mu>1$ and $\lambda_{1}<A$, then the problem (1.1) possesses at least one non-trivial solution pair $z=(u, v) \in$ E. Furthermore, problem (1.1) possesses the least energy non-trivial solution pair $z=(u, v) \in E$.

We will use Mountain Pass theorem to prove Theorem 1.1. As a by-product, we show that

$$
I^{\infty}=\inf \left\{I(z): I^{\prime}(z)=0, z=(u, v) \in E \backslash\{0\}\right\}
$$

is achieved by some $z_{0}=\left(u_{0}, v_{0}\right)$ with $u_{0} \not \equiv 0, v_{0} \not \equiv 0$.

Theorem 1.1 will be proved in Section 2.

2. Existence results. Suppose in this section $\lambda, \mu$ satisfies $\lambda \mu>1$ and $\lambda_{1}<A$. By $\left(H_{1}\right)-\left(H_{3}\right)$, it is easy to see that there is a $2<p<2 N /(N-2)$ if $N>2$ and $2<$ $p<+\infty$ if $N \leq 2$ and that for any $\epsilon>0$ there is a $c_{\epsilon}>0$ such that for $\forall(x, t) \in \Omega \times \mathbb{R}$,

$$
|f(x, t)|,|g(x, t)| \leq \epsilon|t|+c_{\epsilon}|t|^{p-1} .
$$


So the corresponding energy function

$$
I(u, v)=\frac{1}{2}\|z\|^{2}-\int_{\Omega} F(x, u) d x-\int_{\Omega} G(x, v) d x
$$

is well defined on $E$ and class $C^{1}(E, \mathbb{R})$, where $F(x, t)=\int_{0}^{t} f(x, s) d s$ and $G(x, t)=$ $\int_{0}^{t} g(x, s) d s$. Moreover, the Fréchet derivative $I^{\prime}$ satisfying

$$
\begin{aligned}
\left\langle I^{\prime}(u, v),(\varphi, \psi)\right\rangle= & \int_{\Omega}[\nabla u \nabla \psi+\nabla v \nabla \varphi+\lambda \nabla u \nabla \varphi+\mu \nabla v \nabla \psi] d x \\
& -\int_{\Omega} f(x, u) \varphi d x-\int_{\Omega} g(x, v) \psi d x
\end{aligned}
$$

for $\forall(\varphi, \psi) \in E$.

Sequence $\left\{z_{n}\right\} \subset E$ is called the Palais-Smale sequence of a $C^{1}$ function $I$ on $E$ at level $c\left((P S)_{c}\right.$-sequence for short) if $I\left(z_{n}\right) \rightarrow c$ and $I^{\prime}\left(z_{n}\right) \rightarrow 0$ as $n \rightarrow \infty$. To get a $(P S)_{c}$-sequence, we will use the Mountain Pass theorem cited in [8].

Proposition 2.1. Let $E$ be a Hilbert space, $I \in C^{1}(E, \mathbb{R}), e \in E$ and $r>$ 0 such that $\|e\|>r$ and $b:=\inf _{\|z\|=r} I(z)>I(0) \geq I(e)$. Let $c$ be characterised by $c:=\inf _{\gamma \in \Gamma} \max _{\tau \in[0,1]} I(\gamma(\tau))$, where $\Gamma:=\{\gamma \in C([0,1], E): \gamma(0)=0, \gamma(1)=e\}$. Then, there exists a sequence $\left\{z_{n}\right\} \subset E$ such that $I\left(z_{n}\right) \rightarrow c$ and $I^{\prime}\left(z_{n}\right) \rightarrow 0$ as $n \rightarrow \infty$.

LEMmA 2.1. Let $\left(H_{1}\right)-\left(H_{3}\right)$ hold. Then we have the following:

(a) There exist $\rho, \beta>0$ such that $I(z) \geq \beta$ for all $z \in E$ with $\|z\|=\rho$.

(b) There exists $e \in E$ with $\|e\| \geq \beta$ such that $I(e)<0$.

Proof. (a) It follows from (2.1) and the Sobolev embedding theorem that for any $\epsilon>0$ there is a $c_{\epsilon}>0$ such that

$$
\int_{\Omega} F(x, u) d x+\int_{\Omega} G(x, v) d x \leq c \epsilon\|z\|^{2}+c_{\epsilon}\|z\|^{p}
$$

for all $z=(u, v) \in E$. This, jointly with (2.2) implies (a).

(b) By Fatou's Lemma, we have

$$
\begin{aligned}
\lim _{t \rightarrow \infty} \frac{I\left(t \varphi_{1}, t \varphi_{1}\right)}{t^{2}} & =\frac{1}{2} \int_{\Omega}(2+\lambda+\mu)\left|\nabla \varphi_{1}\right|^{2} d x-\lim _{t \rightarrow \infty} \int_{\Omega} \frac{F\left(x, t \varphi_{1}\right)+G\left(x, t \varphi_{1}\right)}{t^{2}} d x \\
& \leq \frac{1}{2} \int_{\Omega}(2+\lambda+\mu)\left|\nabla \varphi_{1}\right|^{2} d x-\int_{\Omega} \lim _{t \rightarrow \infty} \frac{F\left(x, t \varphi_{1}\right)+G\left(x, t \varphi_{1}\right)}{t^{2} \varphi_{1}^{2}} \varphi_{1}^{2} d x \\
& =\frac{1}{2} \int_{\Omega}(2+\lambda+\mu)\left|\nabla \varphi_{1}\right|^{2} d x-\frac{1}{2} \int_{\Omega}(l+m) \varphi_{1}^{2} d x \\
& =\frac{1}{2}\left(2+\lambda+\mu-\frac{l+m}{\lambda_{1}}\right) \int_{\Omega}\left|\nabla \varphi_{1}\right|^{2} d x<0
\end{aligned}
$$

because of $\lambda_{1}<A$. So $I\left(t \varphi_{1}, t \varphi_{1}\right) \rightarrow-\infty$ as $t \rightarrow \infty$ and part $(b)$ is proved. 
Proposition 2.2. If $(u, v) \in H_{0}^{1}(\Omega) \times H_{0}^{1}(\Omega)$ is a non-trivial solution of (1.1), then we have $\lambda_{1} \leq \frac{m \lambda+\mu l-\sqrt{(m \lambda-\mu l)^{2}+4 m l}}{2(\lambda \mu-1)}$.

Proof. Let $k=\frac{\mu l-m \lambda+\sqrt{(m \lambda-\mu l)^{2}+4 m l}}{2 m}$. It is apparent that $(u, v)=(u, k \tilde{v})$ is a nontrivial solution pair of the problem

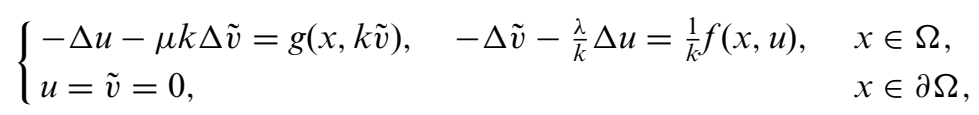

that is

$$
-\left(1+\frac{\lambda}{k}\right) \Delta\left(u+\frac{1+\mu k}{1+\frac{\lambda}{k}} \tilde{v}\right)=g(x, k \tilde{v})+\frac{1}{k} f(x, u) .
$$

By $\left(H_{3}\right)$ and $\left(H_{4}\right)$, we have

$$
\begin{aligned}
\left(1+\frac{\lambda}{k}\right) \int_{\Omega}\left|\nabla\left(u+\frac{1+\mu k}{1+\frac{\lambda}{k}} \tilde{v}\right)\right|^{2} d x & =\int_{\Omega}\left[g(x, k \tilde{v})+\frac{1}{k} f(x, u)\right]\left(u+\frac{1+\mu k}{1+\frac{\lambda}{k}} \tilde{v}\right) d x \\
& \leq \int_{\Omega}\left[m k \tilde{v}+\frac{l}{k} u\right]\left(u+\frac{1+\mu k}{1+\frac{\lambda}{k}} \tilde{v}\right) d x \\
& =\frac{l}{k} \int_{\Omega}\left(u+\frac{m k^{2}}{l} \tilde{v}\right)\left(u+\frac{1+\mu k}{1+\frac{\lambda}{k}} \tilde{v}\right) d x
\end{aligned}
$$

By the definition of $k$ we know that $\frac{1+\mu k}{1+\frac{\lambda}{k}}=\frac{m k^{2}}{l}$, and hence

$$
\lambda_{1} \leq \frac{\frac{l}{k}}{1+\frac{\lambda}{k}}=\frac{l}{k+\lambda}=\frac{m \lambda+\mu l-\sqrt{(m \lambda-\mu l)^{2}+4 m l}}{2(\lambda \mu-1)} .
$$

The proof is complete.

Proposition 2.3. Under assumptions $\left(H_{1}\right)-\left(H_{4}\right)$, problem (1.1) possesses at least one non-trivial solution pair $(u, v) \in E$.

Proof. Proposition 2.1 and Lemma 2.1 implies that there exists a $(P S)_{c}$-sequence $\left\{z_{n}\right\} \subset E$ for $I$, that is

$$
I\left(z_{n}\right) \rightarrow c, \quad I^{\prime}\left(z_{n}\right) \rightarrow 0,
$$

where $c>0$. To get a non-trivial solution, we only need to show that $\left\{z_{n}\right\}$ is bounded in $E$. For this purpose, we suppose, by contradiction, that $\left\|z_{n}\right\| \rightarrow \infty$ as $n \rightarrow \infty$ and let

$$
t_{n}=\frac{2 \sqrt{c}}{\left\|z_{n}\right\|}, \quad w_{n}=t_{n} z_{n}=\frac{2 \sqrt{c} z_{n}}{\left\|z_{n}\right\|}=\left(\frac{2 \sqrt{c} u_{n}}{\left\|z_{n}\right\|}, \frac{2 \sqrt{c} v_{n}}{\left\|z_{n}\right\|}\right) \triangleq\left(w_{n}^{1}, w_{n}^{2}\right) .
$$

Obviously, $\left\{w_{n}\right\}$ is bounded in $E$. By extracting a sub-sequence, we may suppose that

$$
w_{n} \rightarrow w \in E, \quad w_{n} \rightarrow w \text { a.e. in } \Omega
$$

as $n \rightarrow \infty$, where $w=\left(w_{1}, w_{2}\right)$. 
We claim that

$$
w \not \equiv 0 \text {. }
$$

In fact, by $\left(H_{2}\right)-\left(H_{4}\right)$, we see that there exists $M>0$ such that $|f(x, t) / t| \leq M$, $|g(x, t) / t| \leq M$ for all $x \in \Omega$ and $t \geq 0$. Supposing $w \equiv 0$, by Sobolev embedding theorem that, $w_{n}^{1} \rightarrow 0, w_{n}^{2} \rightarrow 0$ in $L^{2}(\Omega)$, as $n \rightarrow \infty$. Then it follows from (2.4) and (2.5) that

$$
\begin{aligned}
4 c & =\int_{\Omega}\left[\frac{f\left(x, u_{n}\right)}{u_{n}}\left|w_{n}^{1}\right|^{2}+\frac{g\left(x, v_{n}\right)}{v_{n}}\left|w_{n}^{2}\right|^{2}\right] d x+o(1) \\
& \leq M \int_{\Omega}\left[\left|w_{n}^{1}\right|^{2}+\left|w_{n}^{2}\right|^{2}\right] d x+o(1) \rightarrow 0
\end{aligned}
$$

as $n \rightarrow \infty$, which is impossible as $c>0$. Hence, the claim is proved.

Set

$$
p_{n}(x)=\left\{\begin{array}{ll}
\frac{f\left(x, u_{n}\right)}{u_{n}} & \text { if } u_{n}(x)>0 ; \\
0 & \text { if } u_{n}(x) \leq 0,
\end{array} \quad q_{n}(x)= \begin{cases}\frac{g\left(x, v_{n}\right)}{v_{n}} & \text { if } v_{n}(x)>0 \\
0 & \text { if } v_{n}(x) \leq 0\end{cases}\right.
$$

By $\left(H_{2}\right)-\left(H_{4}\right)$, we see that

$$
0 \leq p_{n}(x) \leq l, \quad 0 \leq q_{n}(x) \leq m, \quad \forall x \in \Omega,
$$

and there exist two functions $p(x), q(x) \in L^{\infty}(\Omega)$ such that

$$
p_{n} \rightarrow p, q_{n} \rightarrow q \text { in } L^{2}(\Omega)
$$

as $n \rightarrow \infty$. It results to

$$
p_{n}(x) w_{n}^{1} \rightarrow p(x) \max \left\{w^{1}(x), 0\right\}, q_{n}(x) w_{n}^{2} \rightarrow q(x) \max \left\{w^{2}(x), 0\right\} \text { in } L^{2}(\Omega)
$$

as $n \rightarrow \infty$. Since $\left\{z_{n}\right\}$ is a $(P S)_{c}$-sequence of $I$, then from (2.3) we have $\forall(\varphi, \psi) \in E$, so that

$$
\begin{aligned}
o(1)= & \int_{\Omega}\left[\nabla w_{n}^{1} \nabla \psi+\nabla w_{n}^{2} \nabla \varphi+\lambda \nabla w_{n}^{1} \nabla \varphi+\mu \nabla w_{n}^{2} \nabla \psi\right] d x \\
& -\int_{\Omega} p_{n}(x) w_{n}^{1} \varphi d x-\int_{\Omega} q_{n}(x) w_{n}^{2} \psi d x .
\end{aligned}
$$

Letting $n \rightarrow \infty$, we obtain

$$
\begin{aligned}
& \int_{\Omega}\left[\nabla w^{1} \nabla \psi+\nabla w^{2} \nabla \varphi+\lambda \nabla w^{1} \nabla \varphi+\mu \nabla w^{2} \nabla \psi\right] d x-\int_{\Omega} p(x) \max \left\{w^{1}, 0\right\} \varphi d x \\
& -\int_{\Omega} q(x) \max \left\{w^{2}, 0\right\} \psi d x=0 .
\end{aligned}
$$

Therefore, $w^{1}$ and $w^{2}$ satisfy

$$
\left\{\begin{array}{l}
-\Delta w^{1}-\mu \Delta w^{2}=q(x) \max \left\{w^{2}, 0\right\} \geq 0, x \in \Omega, \\
-\Delta w^{2}-\lambda \Delta w^{1}=p(x) \max \left\{w^{1}, 0\right\} \geq 0, x \in \Omega .
\end{array}\right.
$$


Choosing $\left(\varphi_{1}, 0\right)$ as a test function in (2.6), we can get that

$$
\int_{\Omega}\left[\nabla w^{2} \nabla \varphi_{1}+\lambda \nabla w^{1} \nabla \varphi_{1}\right] d x=\int_{\Omega} p(x) \max \left\{w^{1}, 0\right\} \varphi_{1} d x=l \int_{\Omega \cap\left\{x: w^{1}(x)>0\right\}} w^{1} \varphi_{1} d x,
$$

but

$$
\int_{\Omega}\left[\nabla w^{2} \nabla \varphi_{1}+\lambda \nabla w^{1} \nabla \varphi_{1}\right] d x=\int_{\Omega}\left[\lambda_{1} w^{2} \varphi_{1}+\lambda \lambda_{1} w^{1} \varphi_{1}\right] d x
$$

thus we have

$$
\int_{\Omega \cap\left\{x: w^{1}(x)>0\right\}}\left(l-\lambda \lambda_{1}\right) w^{1} \varphi_{1} d x \leq \int_{\Omega \cap\left\{x: w^{2}(x)>0\right\}} \lambda_{1} w^{2} \varphi_{1} d x .
$$

Similarly, choosing $\left(0, \varphi_{1}\right)$ as a test function in (2.6), we can get

$$
\int_{\Omega \cap\left\{x: w^{2}(x)>0\right\}}\left(m-\mu \lambda_{1}\right) w^{2} \varphi_{1} d x \leq \int_{\Omega \cap\left\{x: w^{1}(x)>0\right\}} \lambda_{1} w^{1} \varphi_{1} d x .
$$

If $\Omega \cap\left\{x: w^{2}(x)>0\right\}=\emptyset$, then from (2.7) we know that the maximum principle implies that $w^{1}=-\mu w^{2} \geq 0$ in $\Omega$, but $w=\left(w_{1}, w_{2}\right) \not \equiv 0$, so we must have $\Omega \cap\{x$ : $\left.w^{1}(x)>0\right\} \neq \emptyset$. Hence we can conclude from (2.8) that $l-\lambda \lambda_{1} \leq 0$, which contradicts $\lambda_{1}<A$. Therefore $\Omega \cap\left\{x: w^{2}(x)>0\right\} \neq \emptyset$. Similarly, we have $\Omega \cap\left\{x: w^{1}(x)>0\right\} \neq$ $\emptyset$. Thus, combining (2.8) and (2.9), we can get

$$
\left(l-\lambda \lambda_{1}\right)\left(m-\mu \lambda_{1}\right) \leq \lambda_{1}^{2},
$$

which is impossible since $\lambda_{1}<A$.

Thus, we must have $\left\|z_{n}\right\| \leq c<+\infty$ and the Proposition is proved.

The proof for Theorem 1.1 will be completed by the following Proposition.

Proposition 2.4. If $\left(H_{1}\right)-\left(H_{4}\right)$ hold, then $I^{\infty}$ is assumed.

Proof. By Proposition 2.3, we know that $I^{\infty}$ is well defined and finite. Now we show that $I^{\infty}$ is assumed. Using (2.1) and Sobolev embedding theorem, we get

$$
\|z\|^{2}=\int_{\Omega} f(x, u) u d x+\int_{\Omega} g(x, v) v d x \leq \epsilon c\|z\|^{2}+c_{\epsilon}\|z\|^{p} .
$$

When $\epsilon$ is small enough, we have

$$
\|z\| \geq c>0 .
$$

Suppose now $z_{n}=\left(u_{n}, v_{n}\right) \not \equiv 0$ is a minimising sequence of $I^{\infty}$. By Proposition 2.3, we see that $\left\{z_{n}\right\}$ is uniformly bounded in $E$. So we may assume $z_{n} \rightarrow z=(u, v)$ in $E$ and $I^{\prime}(z)=0$. Since (2.10) implies $z \neq(0,0)$, it follows that $I^{\infty}=\lim _{n \rightarrow \infty} I\left(z_{n}\right)=I(z)$. Consequently, $I^{\infty}$ is assumed by $z \in E \backslash\{0\}$. The proof is complete.

Proof of Theorem 1.1. This is a direct consequence of Proposition 2.3 and 2.4.

ACKNOwLEDGEMENT. This work is supported by Natural Science Foundation of South-Central University for Nationalities, yzz08001. 


\section{REFERENCES}

1. Ph. Clement, D. G. Figueiredo and E. Mitidieri, Positive solutions of semilinear elliptic systems, Comm. PDE. 17 (1992), 923-940.

2. D. G. de Figueiredo and P. L. Felmer, On superquadratic elliptic systems, Trans. Amer. Math. Soc. 343 (1994), 99-106.

3. J. Hulshof and R. C. A. M. Van de Vorst, Differential systems with strongly indefinite linear part, J. Funct. Anal. 114 (1993), 32-58. 954.

4. G. Li and J. Yang, Asymptotically linear elliptic system, Comm. PDE. 29 (2004), 925

5. C. Peng and J. Yang, Nonnegative solutions for nonlinear elliptic systems, J. Math. Anal. Appl. 330 (2007), 633-653.

6. C. Peng and J. Yang, Positive solution for asymptotically linear elliptic systems, Glasgow Math. J. 49 (2007), 377-390.

7. R. C. A. M. Van de Vorst, Variational identities and applications to differential systems, Arch. Rational Mech. Anal. 116 (1991), 375-398.

8. M. Willem, Minimax theorems (Birkhauser, Boston, 1996). 